\title{
Research and Design of a New Type of Intelligent Rubbish Bin
}

\author{
LI Yongmei ${ }^{1, a}$, ZHOU Lei ${ }^{2, b}$, ZHANG Weifen ${ }^{3, c}$ \\ ${ }^{1}$ Department of Mechanical Engineering,Chengxian Institute,Southeast University,Nanjing Jiangsu \\ 210088, China \\ ${ }^{2}$ Department of Mechanical Engineering,Chengxian Institute,Southeast University,Nanjing Jiangsu \\ 210088, China \\ ${ }^{3}$ Department of Mechanical Engineering,Chengxian Institute,Southeast University,Nanjing Jiangsu \\ 210088,China \\ aemail:yml_1108@163.com, bemail:149687158@qq.com,cemail:11376714@qq.com
}

\section{Keywords: Wheel Robot; Rubbish Bin; Voice Control; Avoid Obstacles}

\begin{abstract}
With the widely spread of the concept of integrated electronics, intelligentization has become a trend of future home living, thus, rubbish bin, as a kind of common household appliance, also need to be equipped with intelligence. The rubbish bin is designed on the basis of wheel robot, which could move forward or backward and turn left or right by voice command. Ultrasonic sensors are attached on The rubbish bin robot, therefore, it could effectively avoid obstacles while moving. The rubbish bin lid controlled by steering motor can also open or close smoothly by voice control. This intelligent rubbish bin is very suitable for people who move with difficulty, and it will have a great space to make progress as a family service robot.
\end{abstract}

\section{Introduction}

At present, the intelligent rubbish bins which are sold on market have the general character of low intelligence level and limited function. They basically use photoelectric sensors or infrared sensors to detect the human body signal, afterwards drive the DC motor through the microcontroller unit in order to open the lid and then shutdown 5 or 6 seconds later. Their main shortcomings include two points, first, single function and low intelligence, second, unreasonable mechanical design in some products. For instance, a large number of products are driven by DC motor through hinge, however, the connection or fixing between motor shaft and hinge is unreasonable which leads to the reduction of their service life. Aiming at compensating the defects of the existing intelligent rubbish bin products, adding voice control and obstacle avoidance function based on wheel robot could actually improve the intelligence level and the human-machine interaction of the rubbish bin[1] [2].

\section{Structure of the whole system}

This design of intelligent rubbish bin is based on AVRmega328P-PU MCU. This paper mainly introduces the robot mechanical structure, the hardware circuit and its realization of the control part. The structure of the rubbish bin robot system is shown in figure 1 .

Mechanical structure part: The mechanical structure part of rubbish bin robot is the mobile mechanism, in other words, the body of the robot which loads the walking mechanism and control circuit.

Hardware control circuit part: The design of the robot is based on voice control in this paper.

\section{Mechanical structure}

Rubbish bin robot services in the indoor environment, it is a little bit bigger than ordinary trash can. This robot is mainly used in home or office and it could be easy to replace garbage bags. The structures of the rubbish bin robot form top to bottom are as follows: lid, body, chassis. The lid divides in two parts: a removable cover and an automatic top lid. The removable cover is 
designed for quicker replacing and assembling of garbage bags. The size of the top lid is smaller than bin cover, and it is embedded on the cover. [3] The cover and the lid are connected by specialized support mechanism, besides, the cover and the body are connected by a hinge. In order to make assembly and wiring more easier, the barrel body has two layers, the outer one is the bin shell and the inner layer actually provides space to store rubbish. The rubbish bin robot uses wheel structure to make sure it could walk forward or backward, turn left or right flexibly. The power and control components are installed on the chassis and so do the driving wheels and auxiliary universal wheels, which could not only lower the barycenter to increase the stability while moving but also make the electronic components could be unified installed and avoid the circumstance that components separate too far away from each other [4]. The robot entity is shown in figure 2.

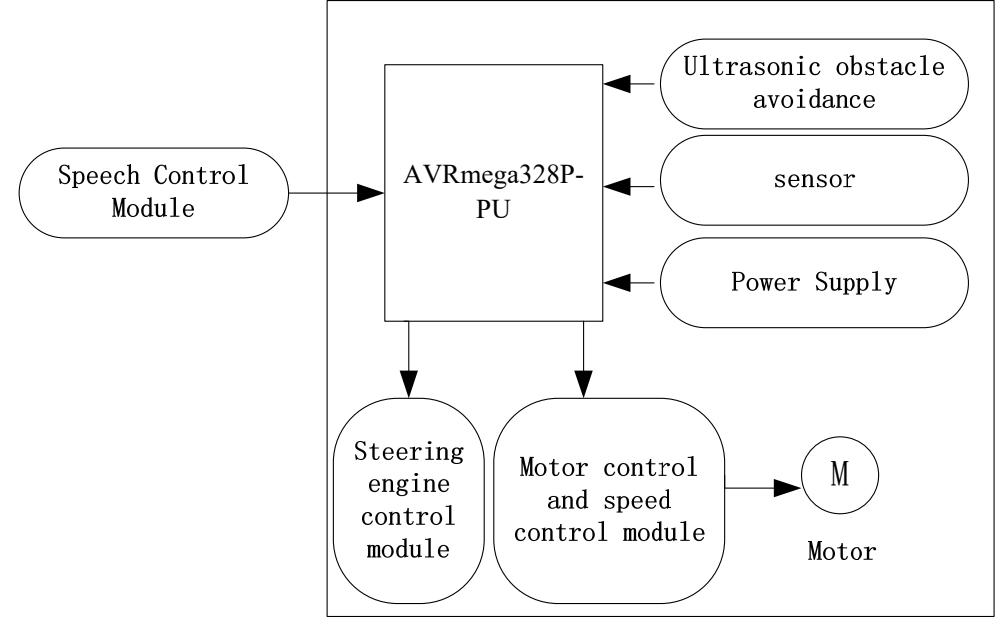

Figure 1, Principle Block Diagram

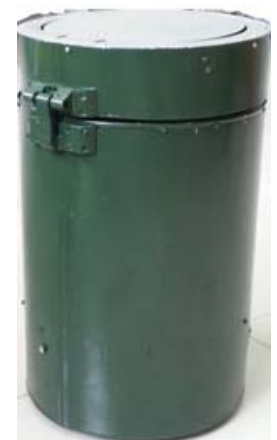

Figure 2, Physical map

1、 configuration of power source

The rubbish bin robot has two motors, one acts the role of main motor which controls the robot walk and the other one control the lid open or close. Due to the weight of the rubbish bin should be taken in to consideration while selecting main motor, it is important to ensure that the motor has enough drag force. There has the same problem in choosing the cover-opening motor, it is not wise to open the lid by controlling DC motor with delay program because the DC motor does not have enough torque and it is easy to make the current overload even burn out the driver board. In summary, $12 \mathrm{~V}$ high power DC motor is chosen to drive the wheels on the chassis in this design and steering motor, under the assistance of special-design bracket, controls the barrel lid open or close. DC motor converts the DC electric power into mechanical energy which has a large start torque and a good performance of speed control.

2、 structure design of wheels on the chassis

At the bottom there are two driving wheels which are distributed in opposite directions and three universal wheels which are designed to adjust the robot turning[5]. Two wheels are driven by two independent motors, but universal wheels does not provide power. Motors, wheels, universal wheels and controllers, circuit boards are all setup on the chassis as shown in figure 3. 


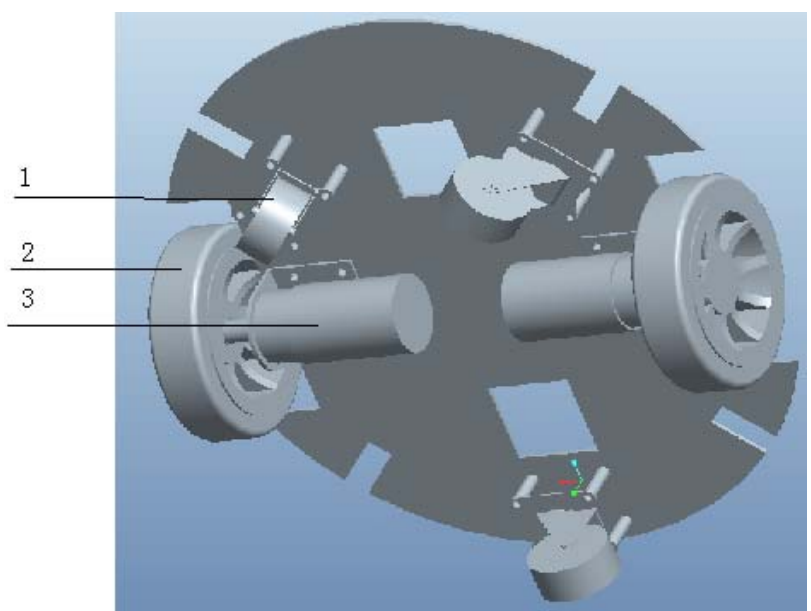

Figure 3, 3D structure of wheels

1.universal wheels 2.driving wheels 3.motors

3、 whole mechanical structure design

With the exception of wheels, the front end of of the chassis is equipped with ultrasonic sensors for obstacle avoidance. The circuit board and the container are on the above of the chassis, besides, on the top of the robot is the voice output module and the lid. For the sake of replacing garbage bag easily, the lid has two layers, one for manual opening to replace garbage bag as well as charging the batteries on the top, the other one is automatically opened by motor when it is in use.

1)chassis

Material: acrylic plate; diameter of outer circle: $290 \mathrm{~mm}$; thickness: $2 \mathrm{~mm}$, as shown in figure 4 .

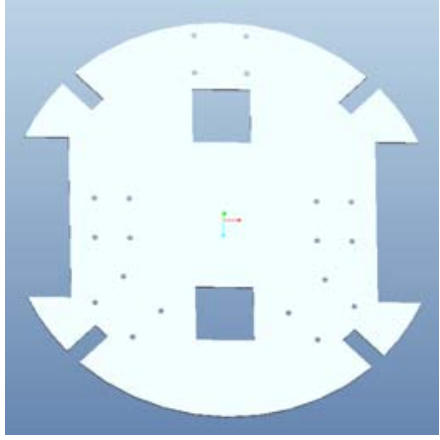

Figure 4, design of chassis

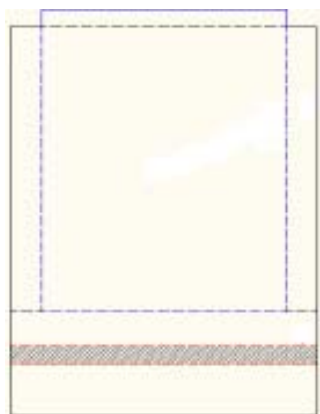

Figure 5, schematic diagram of barrel body structure

\section{2)barrel body}

The body is divided into two parts: inner barrel and outer barrel. In the front of the top of outer barrel has a visible space which could setup ultrasonic distance measuring sensor, the structure is shown in figure 5. Material: engineering plastic; height: $400 \mathrm{~mm}$; diameter of outer circle: $300 \mathrm{~mm}$; garbage storage area diameter $266 \mathrm{~mm}$; depth: $280 \mathrm{~mm}$; material thickness: $2 \mathrm{~mm}$.

3)lid

Barrel lid has two parts: top lid and the cover. The cover is connected with outer barrel by hinge. In addition, top lid is controlled by steering motor under the assistance of self-design bracket. The lid components are shown in figure 6.
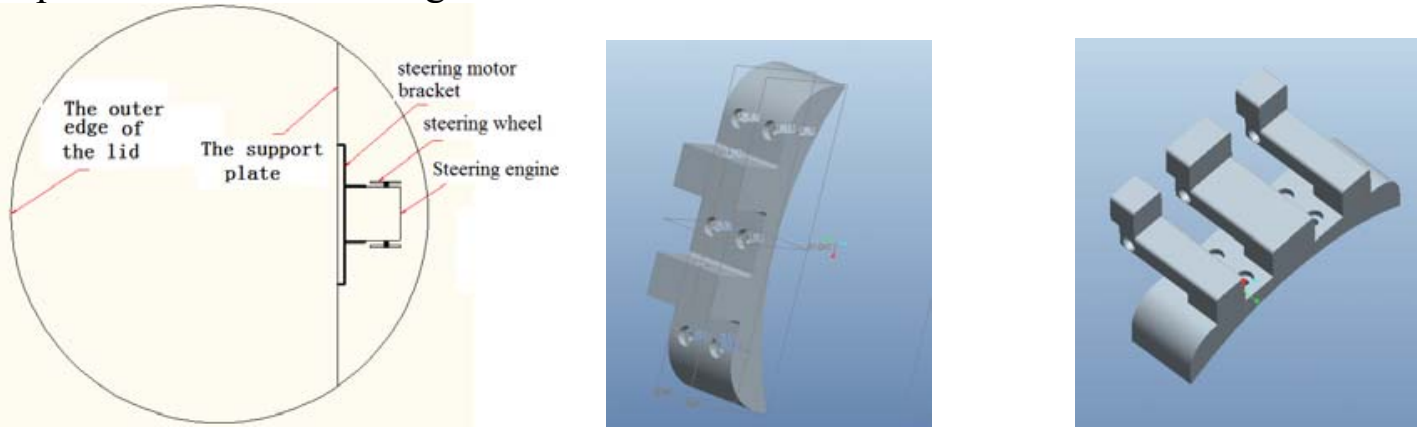
a

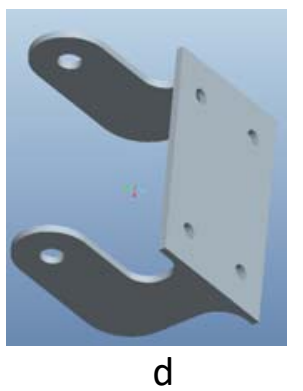

b

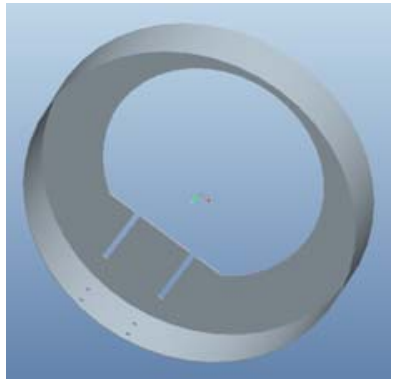

e
C

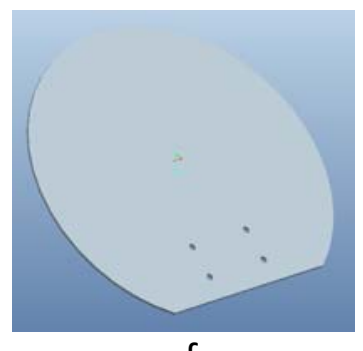

f

Figure 6, components of lid

4)detection structure
a. schematic diagram of lid
b. hinge
c. hinge
d. steering motor bracket
e. cover
f. top lid

On the bottom of the outer barrel, there is a groove which provides a visible detection space for ultrasonic distance sensor. The rubbish bin robot judges whether there are obstacles in front of it by ultrasonic distance sensor in order to determine whether it could go straight on or make a turn to avoid obstacles. The visible window on the barrel is shown in figure 7 .

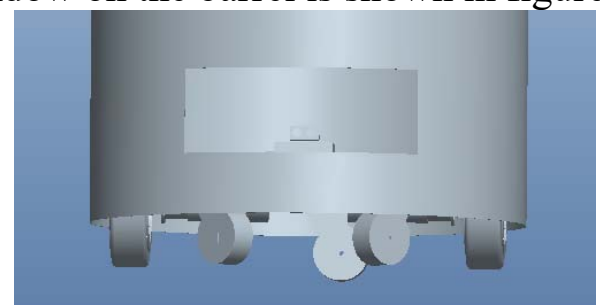

figure 7, visible window for ultrasonic detection

\section{Hardware design of control system}

Three requirements of controlling:

1)Human-machine interaction can be realized, for example, human give orders by voice and the robot provides corresponding actions (walk forward or backward, turn around etc.) instead of manual operating by remote control;

2)It has the function of obstacle avoidance. Because of the complex environment and inevitable obstacles in practical applications, rubbish bin robot has to have the ability to effectively avoid the obstacles.

3)After the system activated, users only need to issue voice orders, other actions are automatically carried out by system.

Based on the requirements above, the hardware of control circuit includes Arduino uno r3CPU control module, voice recognition module, voice synthesis module, 433 wireless module, ultrasonic detection module, I/O extension module, L298N motor driver module, decompression module and power expansion module. Each module is designed independently, all of which have standard interface, and they can be freely combined in order to develop various design solutions flexibly. The design target of hardware is that the power supply can drive the motor and the steering motor, besides, it can also provide energy to the ultrasonic distance sensor, the main controller and the voice command module. The main controller must control the rubbish bin exactly and effectively. The sensor recognizes the obstacle in front of the robot and provides interrupt signal for system to avoid the obstacle. In addition, the robot needs some modules about voice control such us voice receiving module and voice processing module, which are able to translate the human voice command into the signal that can be identified by the controller.

\section{1、 MCU control module}

The circuit hardware in this paper selects AVR mega328P-PU MCU made by Atmel Company, whose parameters are as follow: ATMEGA32x processor series; AVR8 core; 8-bit-wide data bus; 
$32 \mathrm{kB}$ flash ROM etc. Arduino controller mainly made up of mega328P-PU MCU is an integrated circuit board which has an IDE integrated development environment of using high-level language like JAVA or C, and it is based on the open source code of USB interface and simple I/O port board.

2、 voice and wireless module

It is necessary to setup on the host computer software before using voice recognition module. Inputting the Chinese instructions in the form of phonetic symbol on the host computer could receive a hexadecimal value according to the sequence of orders. For example, "go forward" could be typed in the form of " a0qian jin $\} "$, besides, the return vale of the first order is " $00 "$ ". Considering the limit storage space of the module, this paper uses about 50 voice instructions in total[6].

The axiom of the voice synthesis module is the same as the voice recognition module, and it is also setup on the host computer. The synthesis module has function generator so it could achieve code by inputting Chinese voice hints. For instance, the robot says "Intelligent robot is at your service, please give an order" when it is turned on, the code of that hint could be put into the program or be called directly.

3、 ultrasonic detection module

Ultrasonic distance sensor is developed and manufactured based on the characteristics of ultrasonic. Ultrasonic itself is a kind of mechanical wave whose frequency is higher than the vibration of acoustic wave. Energy transfer chip can generate ultrasonic wave under the excitation of voltage. Ultrasonic wave has strong penetrability in liquid and solid, especially in the opaque solid, it can penetrate tens of meters in depth. When ultrasonic wave encounters an impurity or interface, it will produce a obvious reflection wave, besides, when it is faced with moving objects, it has Doppler effect. The ultrasonic wave has the characteristics of high frequency, short wavelength and small diffraction phenomenon, in addition, it has good directivity so that it could directional propagate in the form of rays. Therefore, ultrasonic wave is widely used in industry, national defense, and biomedical. Usually, people like to use ultrasonic transducers or ultrasonic probes as an alias for ultrasonic ranging sensors.

\section{Software Design and Experimental Result}

According to the motion control requirements of the whole intelligent robot, the power supply provides the energy and the motors drive the wheels and make the lid open or close. While receiving voice instructions, the controller controls all the movements. This paper respectively writes the main program and subprogram includes forward, backward, turn left, turn right, stop, open and close program according to the process. Through testing and optimization, the rubbish bin robot performs well in the room under the voice command. The flowchart of the control system is shown in figure 8 .

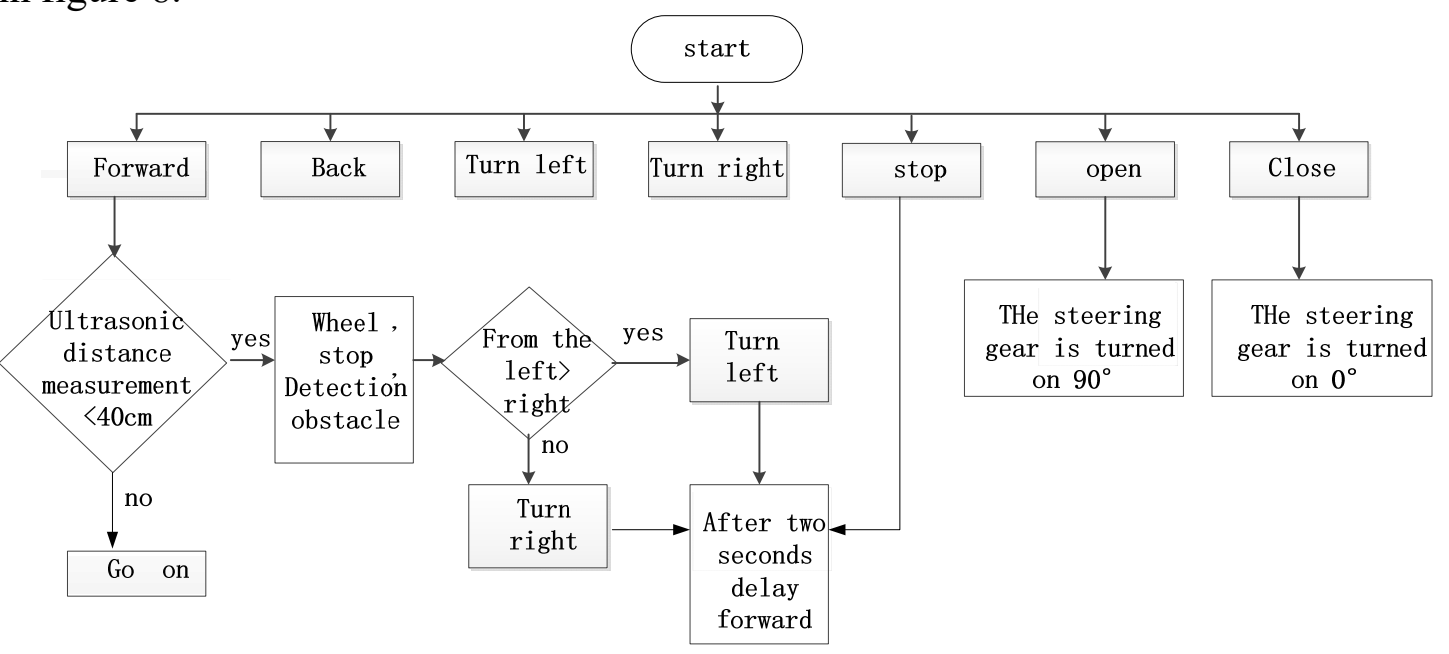

Figure8, flowchart of the control system 


\section{Conclusion}

This paper designed a intelligent rubbish bin robot which can be controlled by voice command. This rubbish bin needs to realize the motion of walking forward or backward, turn left or right, opening or closing the lid only by human voice instructions. In the controllable range, when the owner gives an order, the rubbish bin robot can execute a corresponding action. So, this is a design which combines mechanical, electronic and automation.

\section{References}

[1]WU Guobin, LI Bin, YAN Jizhou. Kinect Human Computer Interaction Development Practice,M, People's Posts and Telecommunications Press,2013.

[2] Alan Winfield, Robotics: A Very Short Introduction, M, Oxford University Press,2012.

[3]Joe Jones, Daniel Roth. Robot Programming :A Practical Guide To Behavior-based Robotics,M,.McGraw-Hill Professional,2013.

[4]Massimo Banzi .Getting Started With Arduino ,M, O’Reilly media ,Inc ,USA ,2011.

[5]David Cook .Intermediate Robot Building,M,APress ,2010.

[6]CHEN Xuedong, JIA Wenchuan. Motion Planning and Control. M, Huazhong University of Science and Technology Press,2006. 